\title{
A Measurement of Beauty Photoproduction Through Decays to Muons and Jets at HERA-II
}

\author{
Benno List* for the H1 Collaboration \\ University of Hamburg - Institute for Experimental Physics \\ Luruper Chaussee 149, D-22603 Hamburg - Germany
}

\begin{abstract}
The photoproduction of beauty quarks in ep collisions has been measured using a data sample of $170 \mathrm{pb}^{-1}$ collected with the H1 detector at HERA-II in the years 2006 and 2007. Events with two jets and a muon in the final state were investigated, and beauty events were identified using the muon's relative transverse momentum to a jet and its impact parameter. Visible cross sections were measured differentially in the transverse momenta of the highest energy jet $\left(p_{\mathrm{T}}^{\text {jet1 }}\right)$ and the muon $\left(p_{\mathrm{T}}^{\mu}\right)$, the pseudorapidity of the muon $\left(\eta^{\mu}\right)$ and of the photon's momentum fraction $x_{\gamma}^{\text {obs }}$ entering the hard interaction. The measurements are found to be well described by QCD calculations at NLO.
\end{abstract}

\section{Introduction}

The production of beauty quarks in ep collisions has been investigated in considerable detail at HERA-I. In several analyses it was observed that measured cross sections where significantly above the predictions from perturbative QCD calculations in next-to-leading order (NLO). The data from HERA-II with its larger statistics makes it possible to repeat these measurements with increased accuracy.

This measurement 1, 2 follows closely a measurement performed with data from HERAI [3], where beauty photoproduction events were investigated with two jets and a muon in the final state. The result of the HERA-I measurement was that NLO calculations describe the data reasonably well, except for the lowest bin of the muon and jet transverse momentum, $p_{\mathrm{T}}^{\mu}$ and $p_{\mathrm{T}}^{\mathrm{jet} 1}$, where the data were significantly above the predictions. Similar measurement have also been made by the ZEUS collaboration [4, in a slightly different phase space. Here, good agreement was found with QCD predictions, also at low jet and muon $p_{\mathrm{T}}$.

The measurement presented here uses the HERA-II data set to measure the same cross sections in the same visible range as in the previous $\mathrm{H} 1$ publication with increased statistics and correspondingly smaller errors. The data was collected with the H1 detector in the years 2006 and 2007, when HERA collided electron and positron beams of an energy of $E_{\mathrm{e}}=27.55 \mathrm{GeV}$ with protons of $E_{\mathrm{p}}=920 \mathrm{GeV}$, and corresponds to an integrated luminosity of $170 \mathrm{pb}^{-1}$.

\section{QCD Models}

The Monte Carlo generators PYTHIA 6.2 [5] and Cascade 2.0 [6] were used for the simulation of signal and background distributions.

The PYTHIA event samples were generated with massless matrix elements, using the CTEQ6L [7] and SAS-1D [8] parton density sets for the proton and the photon, respectively.

${ }^{*}$ Supported by the German Federal Ministry of Science and Technology under grant 05H16GUA.

DIS 2009 
The fragmentation of heavy quarks to hadrons was simulated using the Peterson fragmentation function with a parameter $\epsilon_{\mathrm{b}(\mathrm{c})}=0.0069(0.058)$ for beauty (charm) quarks. More details on the parameter settings can be found in our previous publication [3].

For cross checks, additional Monte Carlo samples were generated using the Cascade program, which is based on $k_{\mathrm{T}}$ factorization and the CCFM evolution rather than collinear factorization and DGLAP evolution. The proton parton density set A0 [9] is used for the unintegrated gluon density in the proton.

Both programs, PYTHIA and Cascade, use Leading Order (LO), i.e. $O\left(\alpha_{\mathrm{s}}\right)$, QCD matrix elements for the hard scattering, augmented by parton showers that approximate the effect of additional multiple gluon emission.

To compare the data to Next-to-Leading Order (NLO) QCD, i.e. $O\left(\alpha_{\mathrm{s}}^{2}\right)$, the FMNR program [10] has been used, which is based on the NLO calculation by Nason, Dawson, and Ellis [1]. Here, the CTEQ5F4 [12] (GRV-G HO [13]) parton density set for the proton (photon) were employed. Hadronisation corrections were calculated using the PYTHIA program.

The theory uncertainties were determined by varying the input beauty mass $m_{\mathrm{b}}$ from the nominal value $m_{\mathrm{b}}=4.75 \mathrm{GeV}$ up and down by $0.25 \mathrm{GeV}$. In addition, the renormalization and factorization scales $\mu_{\mathrm{r}}$ and $\mu_{\mathrm{f}}$ were varied independently in the range $\mu_{0} / 2 \leq \mu_{\mathrm{r}}, \mu_{\mathrm{f}} \leq$ $2 \mu_{0}$, with the constraint $1 / 2 \leq \mu_{\mathrm{r}} / \mu_{\mathrm{f}} \leq 2$, and $\mu_{0}$ set to $\mu_{0}=\sqrt{p_{\mathrm{T}}^{2}+m_{\mathrm{b}}^{2}}$. For each bin on hadron level, the deviations due to the beauty mass variation and the largest deviation due to the scale variation in the upward and downward direction were determined and added in quadrature for the total model uncertainty, following the prescription in [14, p. 406].

\section{Analysis Method}

Events were selected with the following experimental cuts: No electron candidate with an energy above $E>6 \mathrm{GeV}$ is allowed to be found in the detector. The inelasticity $y$, measured from the hadronic activity in the detector, must lie in the range $0.2<y_{\mathrm{h}}<0.8$. Two jets with transverse momenta $p_{\mathrm{T}}^{\text {jet1(2) }}>7(6) \mathrm{GeV}$ for the highest (second highest) $p_{\mathrm{T}}$ jet have to be found within the pseudorapitity range $-2.5<\eta^{\text {jet }}<2.5$. Jets were identified by the inclusive $k_{\mathrm{t}}$ jet algorithm [15] in the $p_{\mathrm{T}}$ recombination scheme, with a distance parameter $R=1.0$. The jet algorithm was applied in the laboratory frame. A muon, identified in the central muon system, with transverse momentum $p_{\mathrm{T}}^{\mu}>2.5 \mathrm{GeV}$ has to be found in a pseudorapidity range $-0.55<\eta^{\mu}<1.1$. The muon has to be associated to one of the two highest energy jets.

To ensure a good muon reconstruction and suppress events with cosmic muons, additional quality cuts were applied to the muon track; in particular, the track must be associated to at least one hit in the central silicon tracker to ensure a precise measurement of the impact parameter $\delta$.

\section{Cross Section Definition and Measurement}

We have measured the cross section of the process $e p \rightarrow e b \bar{b} X \rightarrow e j j \mu X^{\prime}$, i.e. beauty production with the formation of two jets and the subsequent decay of a beauty hadron to a muon. The muon may be produced by a direct semileptonic decay of a beauty hadron, from a cascade decay, where the charm hadron decays semileptonically, or from a $J / \psi$ or $\psi^{\prime}$ decay. The muon must be associated to either of the two highest $p_{\mathrm{T}}$ jets.

DIS 2009 

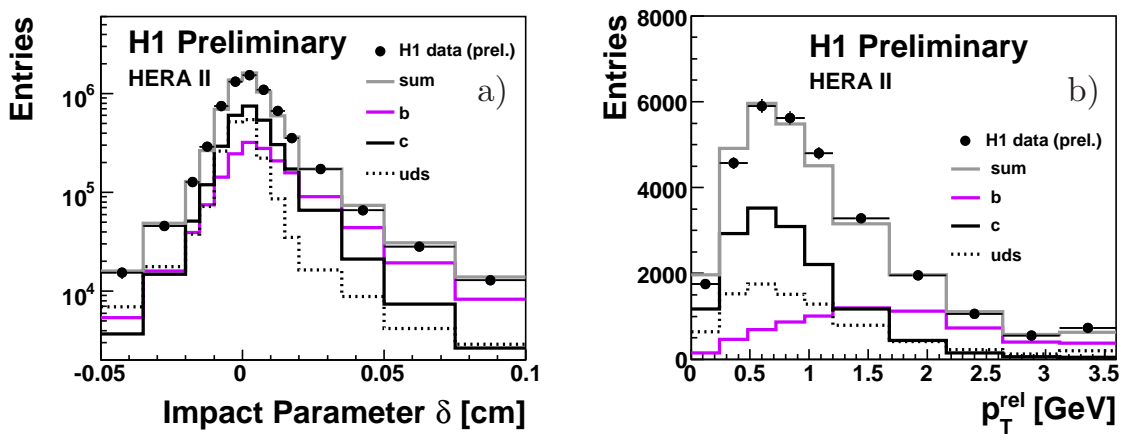

Figure 1: Distributions of a) the impact parameter $\delta$ of the muon track and b) the transverse muon momentum $p_{\mathrm{T}}^{\mathrm{rel}}$ relative to the axis of the associated jet. Included in the figure are the estimated contributions of events arising from beauty quarks (dark grey line), charm quarks (black line) and light quarks (dotted line).

The visible range is defined by $Q^{2}<1 \mathrm{GeV}^{2}, 0.2<y<0.8, p_{\mathrm{T}}^{\mu}>2.5 \mathrm{GeV},-0.55<$ $\eta^{\mu}<1.1 p_{\mathrm{T}}^{\text {jet1(2) }}>7(6) \mathrm{GeV}$, and $-2.5<\eta^{\text {jet1(2) }}<2.5$.

To extract the beauty fraction, two quantities were used: $p_{\mathrm{T}}^{\mathrm{rel}}$, which is the transverse momentum of the muon with respect to the axis of the most energetic jet, reconstructed without the muon four vector, and $\delta$, which is the impact parameter of the muon with respect to the primary vertex of the event. The sign of $\delta$ is determined in relation to the jet axis and defined such that it is positive for muons originating from a secondary vertex displaced along the jet direction.

For each bin, the two-dimensional distribution of these quantities were fitted with three template distributions derived from Monte Carlo simulations, taking into account the statistical uncertainties from the data sample and the Monte Carlo templates [16]; the templates were generated separately for events containing only light $(u, d$, and $s)$ quarks, charm quarks, and beauty quarks, respectively.

The result of the fit is the relative amount of beauty induced events $f_{\mathrm{b}}$ in each analysis bin, from which the observed number of beauty events $N_{\mathrm{b}}$ in the bin is calculated as $N_{\mathrm{b}}=$ $f_{\mathrm{b}} \cdot N_{\text {bin }}$, where $N_{\text {bin }}$ is the total number of data events observed in the respective bin; the data were then corrected for effects of detector resolution by a matrix unfolding procedure, with a migration matrix determined from Monte Carlo simulations.

\section{Control Distributions}

Fig. 1 shows the distributions of the quantities used to extract the beauty fraction from the data: the impact parameter $\delta$ of the muon track and the transverse muon momentum $p_{\mathrm{T}}^{\mathrm{rel}}$ relative to the axis of the associated jet. Both quantities are described quite well by the Monte Carlo simulation. In particular, the impact parameter distribution, which is very sensitive to the detector resolution, is very well described, in the region $\delta<0$ that is dominated by resolution effects as well as in the region $\delta>0$, which shows the tails due to long-lived particles from charm and beauty decays.

DIS 2009 


\section{Systematic Uncertainties}

A number of sources of systematic errors were considered. The overall normalization uncertainty comprises uncertainties on the trigger efficiency, muon identification and track finding efficiencies, and the luminosity. Additional uncertainties that affect the data differently in various bins are: The impact parameter resolution, the reconstruction of the jet axis, the energy scale for hadrons of the calorimeter, the model uncertainties (estimated by using CASCADE [6] instead of PYTHIA 6.2 [5]), the uncertainty from the fragmentation process (estimated by using the Lund instead of the Peterson fragmentation function), the uncertainty from the fragmentation fractions of $c$ and $b$ quarks into hadrons, their branching ratios and lifetimes, and the uncertainty on the modelling of $\pi$ and $K$ inflight decays. The resulting systematic uncertainty is $12 \%$.

\section{Results}

The total cross section for the process $e p \rightarrow e b \bar{b} X \rightarrow e j j \mu X^{\prime}$ in the visible range given above has been measured to be

$$
\sigma_{\mathrm{vis}}\left(e p \rightarrow e b \bar{b} X \rightarrow e j j \mu X^{\prime}\right)=31.4 \pm 1.3(\text { stat. }) \pm 3.8 \text { (syst.) pb. }
$$

This result is somewhat lower than the published result [3] from HERA-I, which is

$\sigma_{\text {vis }}=38.4 \pm 3.4$ (stat. $) \pm 5.4$ (syst.) pb, but compatible within errors. In comparison, the FMNR calculation yields $\sigma_{\text {vis }}=25.3_{-4.7}^{+6.4} \mathrm{pb}$, in agreement with the data, and the PYTHIA prediction is $\sigma_{\text {vis }}=21.7 \mathrm{pb}$.

Differential cross sections were also measured as a function of the following quantities: The transverse momentum of the muon $p_{\mathrm{T}}^{\mu}$, the transverse momentum $p_{\mathrm{T}}^{\text {jet1 }}$ of the highest $p_{\mathrm{T}}$ jet, the pseudorapidity $\eta^{\mu}$ of the muon, $x_{\gamma}^{\text {obs }}$, the momentum fraction of the photon entering the hard interaction, and $\delta \phi_{\text {jets }}$, the difference in azimuthal angle between the two jets.

The differential cross sections also tend to be lower than the HERA-I results, as shown in Fig. 2. The largest discrepancies are observed for the differential measurements in the lowest bins of $p_{\mathrm{T}}^{\mu}$ and $p_{\mathrm{T}}^{\text {jet1 }}$. For these bins, the ratio between the measurements is about $2.5 \sigma$ below unity if systematic uncertainties that do not cancel between both measurements are taken into account. It has been checked that this discrepancy is not caused by differences in the analysis method between the HERA-I and HERA-II analyses; we therefore attribute the difference to a statistical fluctuation.

Fig. 22) shows the difference in azimuthal angle, $\delta \phi_{\text {jets }}$, between the two jets. In leading order QCD the two outgoing quarks must be exactly opposite in azimuthal angle, corresponding to $\delta \phi_{\text {jets }}=180^{\circ}$. Thus, values of $\delta \phi_{\text {jets }}$ substantially lower than $180^{\circ}$ are indicative of the presence of further final state gluons, and therefore this quantity is sensitive to the description of gluon emission. PYTHIA, which employs parton showers to simulate the effect of multiple gluon emission, describes the shape of this observable reasonably well, as does the fixed order calculation of FMNR, which allows at most one hard gluon in the final state.

Overall, the data are reasonably well described in shape by the predictions from PYTHIA, but lie approximatly a factor 1.4 above the PYTHIA prediction. The NLO predictions, derived with the FMNR program, also lie systematically below the data, but also describe the differential distributions well in shape. In particular, the deficiency in the lowest bins of $p_{\mathrm{T}}^{\mu}$ and $p_{\mathrm{T}}^{\mathrm{jet} 1}$ is not substantially larger than in the other bins, in contrast to the findings of the HERA-I analysis. This observation agrees with the results from ZEUS [4].

DIS 2009 

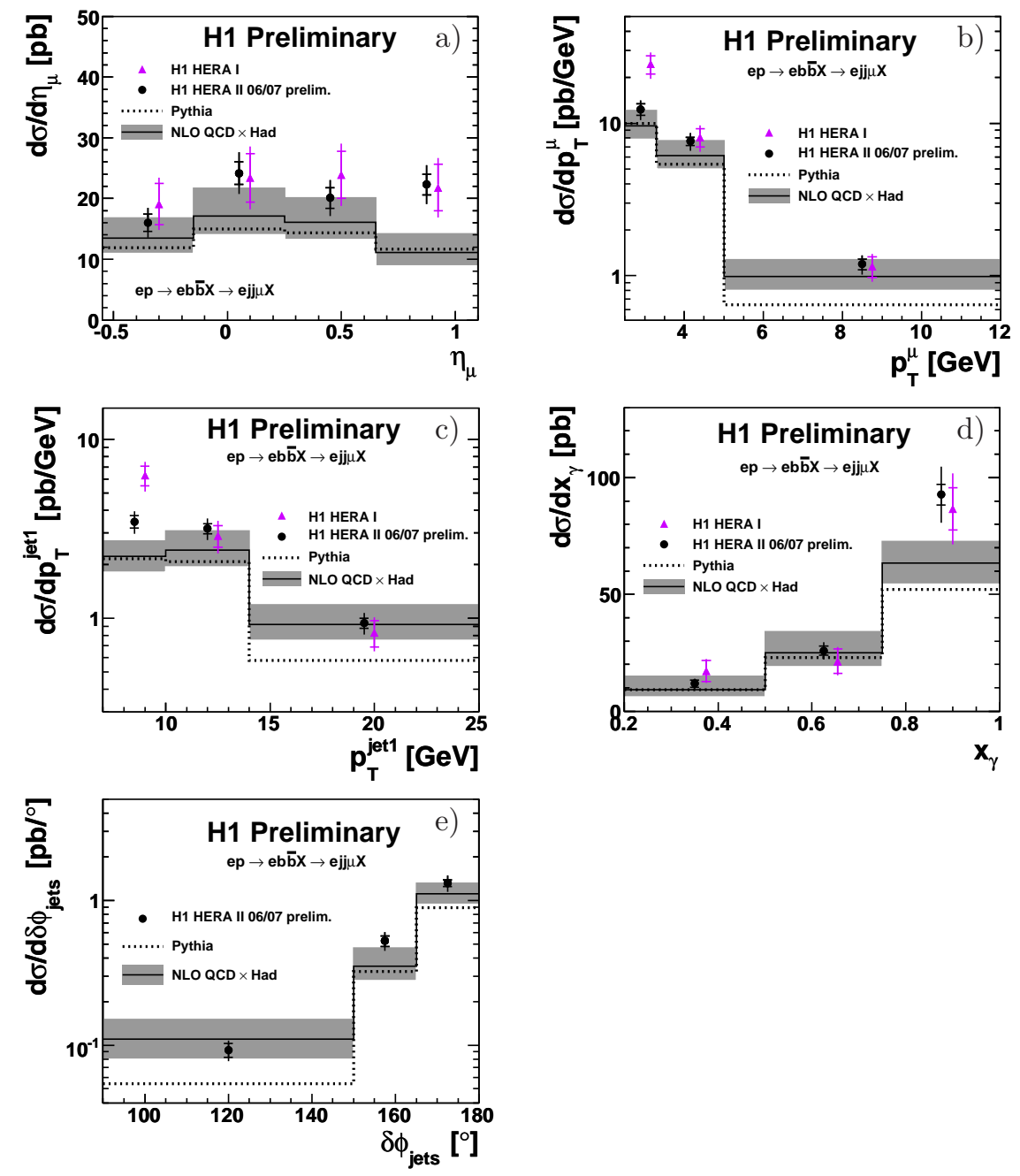

Figure 2: Differential cross sections for the photoproduction process ep $\rightarrow e b \bar{b} X \rightarrow e j j \mu X$ in the kinematic range $Q^{2}<1 \mathrm{GeV}^{2}, 0.2<y<0.8, p_{\mathrm{T}}^{\mu}>2.5 \mathrm{GeV}, 0.55<\eta^{\mu}<1.1, p_{\mathrm{T}}^{\text {jet1(2) }}>$ $7(6) \mathrm{GeV}$ and $\left|\eta^{\text {jet1(2) }}\right|<2.5$. The cross sections are shown as functions of a) the muon pseudorapidity $\eta^{\mu}, \mathrm{b}$ ) the muon transverse momentum $\left.p_{\mathrm{T}}^{\mu}, \mathrm{c}\right)$ the jet transverse momentum $p_{\mathrm{T}}^{\text {jet1 }}$ of the highest transverse momentum jet, d) the photon's momentum fraction $x_{\gamma}^{\text {obs }}$ entering the hard interaction, and e) the azimuthal angle difference $\delta \phi_{\text {jets }}$ between the jets. The inner error bars show the statistical error, the outer error bars represent the statistical and systematic uncertainties added in quadrature. The NLO QCD predictions are corrected to the hadron level (solid line) using the PYTHIA generator. The shaded band around the hadron level prediction indicates the systematic uncertainties as estimated from scale variations (see text). Predictions from the Monte Carlo generator PYTHIA (dotted line) are also shown. 


\section{Conclusions}

We have performed a measurement of the photoproduction of beauty quarks, using events where at least one beauty hadron decays with a muon in the final state, and two jets are visible in the detector, in the phase space region defined by $Q^{2}<1 \mathrm{GeV}^{2}, 0.2<y<0.8, p_{\mathrm{T}}^{\mu}>$ $2.5 \mathrm{GeV},-0.55<\eta^{\mu}<1.1, p_{\mathrm{T}}^{\text {jet1(2) }}>7(6) \mathrm{GeV}$, and $-2.5<\eta^{\text {jet1(2) }}<2.5$.

The visible cross section has been measured to be $\sigma_{\text {vis }}\left(e p \rightarrow e b \bar{b} X \rightarrow e j j \mu X^{\prime}\right)=31.4 \pm$ 1.3 (stat.) \pm 3.8 (syst.) pb. A NLO QCD calculation is in agreement with this measurement within the theoretical uncertainties.

Differential cross sections have been measured as function of the observables $\eta^{\mu}, p_{\mathrm{T}}^{\mu}$, $p_{\mathrm{T}}^{\text {jet1 }}, x_{\gamma}^{\text {obs }}$, and $\delta \phi_{\text {jets. }}$. The shape of these distributions is reasonably well described by the NLO QCD calculation as well as the PYTHIA LO Monte Carlo program.

At low values of $p_{\mathrm{T}}^{\mu}$ and $p_{\mathrm{T}}^{\text {jet1 }}$, the new measurement lies lower than the previous HERA-I measurement published by H1, and is thus better described by the NLO predictions than the previous measurement.

\section{References}

[1] Slides: http://indico. cern. ch/contributionDisplay py? contribId=116\&sessionId=5\&conf Id=53294

[2] H1 Collaboration, H1prelim-08-071 (2008), http://www-h1.desy.de/publications/H1preliminary.short_list.html; M. Krämer, PhD thesis Univ. Hamburg 2009.

[3] A. Aktas et al. [H1 Collaboration], Eur. Phys. J. C41 453 (2005).

[4] S. Chekanov et al. [ZEUS Collab.], Phys. Rev. D70 012008 (2004) [Erratum-ibid. D74 059906 (2006)]; idem, arXiv:0901.2226 [hep-ex] (2009).

[5] T. Sjöstrand et al., Comput. Phys. Commun. 135238 (2001).

[6] H. Jung and G. P. Salam, Eur. Phys. J. C19 351 (2001);

H. Jung, Comput. Phys. Commun. 143100 (2002).

[7] J. Pumplin et al., JHEP 0207012 (2002).

[8] G. A. Schuler and T. Sjöstrand, Phys. Lett. B376 193 (1996).

[9] H. Jung, hep-ph/0411287 (2004).

[10] S. Frixione, P. Nason and G. Ridolfi, Nucl. Phys. B454 3 (1995).

[11] P. Nason, S. Dawson and R. K. Ellis, Nucl. Phys. B303 607 (1988); idem, Nucl. Phys. B327 49 (1989) [Erratum-ibid. B335 260 (1990)]; R. K. Ellis and P. Nason, Nucl. Phys. B312 551 (1989).

[12] H. L. Lai et al. [CTEQ Collaboration], Eur. Phys. J. C12 375 (2000).

[13] M. Glück, E. Reya and A. Vogt, Phys. Rev. D46 1973 (1992).

[14] S. Alekhin et al., hep-ph/0601013 (2006).

[15] S. D. Ellis and D. E. Soper, Phys. Rev. D48 3160 (1993); M. Cacciari and G. P. Salam, Phys. Lett. B641 57 (2006).

[16] R. J. Barlow and C. Beeston, Comput. Phys. Commun. 77219 (1993). 